\title{
Electrical Storm as an Independent Mortality Risk in Patients with Preserved or Moderately Reduced Left Ventricular Function Results from a Japanese Nationwide Registry
}

\author{
Masaya Watanabe, ${ }^{1} \mathrm{MD}$, Hisashi Yokoshiki, ${ }^{2} \mathrm{MD}$, Takashi Noda, ${ }^{3} \mathrm{MD}$, Isao Yokota, ${ }^{4} \mathrm{PhD}$, \\ Takashi Nitta, ${ }^{5}$ MD, Yoshifusa Aizawa, ${ }^{6}$ MD, Tohru Ohe, ${ }^{7}$ MD and Takashi Kurita, ${ }^{8}$ MD
}

\begin{abstract}
Summary
Electrical storm (ES), defined by 3 or more occurrences of ventricular arrhythmias within 24 hours, has been shown to be associated with an increased risk of mortality; however, detailed information remains lacking. We aimed to examine the incidence and determinants of ES and its impact on mortality in patients enrolled in the nationwide implantable cardioverter-defibrillator (ICD) registry.

We studied 1,256 patients (age $65 \pm 12$ years) who had structural heart disease with an ICD. The patients were classified into reduced ejection fraction ( $\mathrm{EF}<35 \%$; $657(52 \%)$ patients) and preserved or moderately reduced $\mathrm{EF}$ ( $\mathrm{EF} \geq 35 \% ; 599$ (48\%) patients).

ES occurred in $49(7 \%)$ and $36(6 \%)$ patients in the $\mathrm{EF}<35 \%$ and $\mathrm{EF} \geq 35 \%$ groups (log-rank $P=0.297$ ) during the median follow-up of 2.3 years. ICD with resynchronization therapy was associated with a lower incidence of ES in patients with $\mathrm{EF}<35 \%$. Non-ischemic heart disease and diuretics were associated with ES in patients with $\mathrm{EF} \geq 35 \%$. During the follow-up, $10 / 49$ (20\%) patients with ES and 80/608 patients (13\%) without ES died in patients with $\mathrm{EF}<35 \%$, while $7 / 36(19 \%)$ patients with $\mathrm{ES}$ and 38/563 patients (7\%) without ES died in those with $\mathrm{EF} \geq 35 \%$. We have created 4 Cox multivariate models. All models showed approximately 2-fold higher hazard ratios in patients with $\mathrm{EF} \geq 35 \%$ compared to $\mathrm{EF}<35 \%$.

Our study showed that the determinants of ES differed between $\mathrm{EF}<35 \%$ and $\mathrm{EF} \geq 35 \%$. The impact of ES for mortality was numerically higher in $\mathrm{EF} \geq 35 \%$ than in $\mathrm{EF}<35 \%$, although a significant interaction was not detected.
\end{abstract}

(Int Heart J Advance Publication) Key words: Ventricular tachycardia, Non-ischemic heart disease, Mortality, Implantable cardioverter-defibrillator

I mplantable cardioverter-defibrillators (ICDs) are lifesavers and have improved mortality rates in patients at risk of sudden death, both in primary and secondary prevention. ${ }^{1,2)}$ However, they are unable to modify the myocardial substrate, which remains susceptible to lifethreatening VAs. ${ }^{2}$ Electrical storm (ES), which is usually defined by 3 or more occurrences of VA within 24 hours, ${ }^{3,4)}$ has emerged as a form of electrical instability and is highly associated with morbidity and mortality. ${ }^{477}$ Recent studies have suggested that ES is not only a clustered VA episode but is also associated with increased non-sudden cardiac mortality, largely due to worsening heart failure $(\mathrm{HF})^{8-10)}$

Although accumulating evidence suggests that ES is associated with subsequent mortality, ${ }^{4.7}$ it has not been examined whether ES shows a homogeneous influence on mortality in patients with different left ventricular (LV) functions. In addition, while ICD for secondary prevention is a relatively recognized risk of $\mathrm{ES},{ }^{7,10,11)}$ the association between ES and other clinical variables such as ejection fraction (EF) or underlying heart diseases remains controversial.

Therefore, we aimed to compare the incidence rate, risk factors, and prognostic impact of ES in patients with reduced and preserved or moderately reduced LV function in the population of the Nippon Storm Study, a prospective observational study conducted at 48 Japanese ICD centers. ${ }^{\text {) }}$

\section{Methods}

Nippon Storm Study: The trial design and results from

From the ${ }^{1}$ Department of Cardiovascular Medicine, Hokkaido University Hospital, Sapporo, Japan, ${ }^{2}$ Department of Cardiovascular Medicine, Sapporo City General Hospital, Sapporo, Japan, ${ }^{3}$ Department of Cardiovascular Medicine, National Cerebral and Cardiovascular Center, Suita, Japan, ${ }^{4}$ Department of Biostatistics, Graduate School of Medicine, Hokkaido University, Sapporo, Japan, ${ }^{5}$ Department of Cardiovascular Surgery, Nippon Medical School, Tokyo, Japan, ${ }^{6}$ Department of Research and Development, Tachikawa Medical Center, Niigata, Japan, ${ }^{7}$ Okayama City Hospital, Okayama, Japan and ${ }^{8}$ Department of Internal Medicine, Faculty of Medicine, Kindai University, Osaka-Sayama, Japan.

This study was partially supported by JSPS KAKENHI Grant Number JP085700004 to Takashi Noda.

Address for correspondence: Masaya Watanabe, MD, Department of Cardiovascular Medicine, Hokkaido University Hospital, North15, West7, Kita-ku, Sapporo, Hokkaido 060-8638, Japan. E-mail: m.watanabe@ huhp.hokudai.ac.jp

Received for publication December 31, 2020. Revised and accepted June 4, 2021.

Released in advance online on J-STAGE November 17, 2021

doi: 10.1536/ihj.20-832

All rights reserved by the International Heart Journal Association. 
the Nippon Storm Study have been published previously. ${ }^{7)}$ In brief, this study was a multicenter prospective observational study that aimed to investigate the incidence and clinical characteristics of patients who developed ES after ICD implantation. The study included 1570 patients who underwent implantation of a new ICD or ICD with resynchronization therapy (CRTD) between October 2010 and July 2012 in one of the 48 Japanese ICD centers. Among the entire population, patients diagnosed with structural heart disease (SHD) were included in this sub-study. Patients were subsequently divided into 2 groups according to their LVEF, as follows: reduced LV function (LVEF < $35 \%$ ) and preserved/moderately reduced LV function (LVEF $\geq 35 \%$ ).

The indication for ICD therapy was determined by the attending cardiologists at each center according to the latest guidelines for ICD implantation. Patients were followed up until the end of July 2014.

The study protocol complied with the Declaration of Helsinki and was approved by the institutional review board of each participating center. All patients provided written informed consent before participating in the study. ICD programming: ICD programming, including the addition of discrimination algorithms, such as PR Logic and Wavelet (Medtronic, Minneapolis, MN), Rhythm ID (Boston Scientific, Marlborough, MA), and Morphology Discrimination plus AV Rate Branch (St. Jude Medical, St. Paul, MN) was conducted at the discretion of the treating physician. Anti-tachycardia therapies were typically set as follows: ventricular fibrillation (VF) zone $>188-200 \mathrm{bpm}$ with at least one train of anti-tachycardia pacing (ATP) before the shock and ventricular tachycardia (VT) zone > 140 to $160 \mathrm{bpm}$ with at least 3 trains of ATP before the shock. The therapies were modified according to the patients' medical history.

Follow-up: The tracking system "Chaser" was constructed, and the treating physicians were encouraged to arrange an appropriate interval of patient visits; a maximum interval of 6 months was allowed to minimize the loss of follow-up data. Data from appropriate and inappropriate ICD interventions as well as patient information, including physical conditions, electrocardiogram results, chest X-rays, and blood examinations, were sent to the data management center in the office of the Japanese Heart Rhythm Society. Follow-up data were collected until October 2014.

Endpoint definitions: The endpoints of the current study were ES and all-cause mortality. ES was defined as the occurrence of at least 3 separate episodes of VT/VF within a 24-hour period, each of those separated by at least 5 minutes and successfully terminated by the ICD, or by an incessant VT lasting more than 12 hours. ${ }^{12)}$ For each episode of ES, the intracardiac electrograms were blindly reviewed by 2 electrophysiologists and adjudicated based on the above definition. Appropriate ICD therapies were divided into ES and non-ES appropriate therapies.

Statistical analyses: Continuous variables are expressed as the mean \pm standard deviation or median with interquartile range (IQR) and were compared using the Student t-test or Mann-Whitney $U$ test as appropriate. Categorical variables are presented as numbers and percentages (\%) and were compared using Fisher's exact test.

Gray's test was performed to consider the competing risk of death to estimate and compare the cumulative incidence of ES between groups. Predictors of ES occurrence were assessed using the Fine-Gray subdistribution hazard model, considering all-cause death as a competing risk. Each variable that achieved $P<0.10$ in the univariate analysis was included in the multivariate model and was adjusted for prespecified covariables according to our previous study (ICD implantation for secondary prevention and QRS width). ${ }^{7}$

The cumulative incidence of death was estimated using the Kaplan-Meier method and compared using the log-rank test between the groups. Multivariate Cox proportional hazards regression analyses were performed to estimate the relationship between ES and mortality, in which ES was included in the models as a time-dependent variable.

The model was adjusted with variables that achieved $P<0.10$ in the univariate Cox regression analysis and had clinically relevant association with mortality (age at implantation, New York Heart Association [NYHA] class $\geq$ III, sinus rhythm, heart rate, LVEF, CRTD implantation, use of beta-blockers, use of angiotensin-converting enzyme inhibitors (ACEI) or angiotensin II receptor blockers (ARB), creatinine, and BNP. Because of the limited number of events, we created several multivariate models using a combination of the above variables. First, age and NYHA class $\geq$ III were included in all the models. Second, to adjust for electrocardiographic parameters, sinus rhythm and heart rate were included in model 1; to adjust for left ventricular function, LVEF and the use of CRTD were included in model 2; to adjust for the pharmacological therapies, the use of beta-blockers and ACEIs or ARBs were included in model 3 ; to adjust for the parameters of blood examination, creatinine and BNP were included in model 4.

All t-tests were 2-sided and statistical significance was set at $P<0.05$. All statistical analyses were performed using JMP Pro ${ }^{\circledR} 12.0$ (SAS Institute, Cary, NC, USA) and EZR (Saitama Medical Center, Jichi Medical University, Saitama, Japan, version 1.32), which is a graphical user interface for R (R Foundation for Statistical Computing, Vienna, Austria, version 3.6.3).

\section{Results}

Patients: Of the 1570 patients enrolled in the study, 289 who were not diagnosed with SHDs and 25 who lacked clinical $(n=7)$ or follow-up $(n=18)$ data were excluded from the analysis. Overall, 1256 patients $(65 \pm 12$ years old, $76 \%$ male, $62 \%$ with non-ischemic heart disease) were included in this study. At the time of implantation, 657 patients showed a reduced LV function while another 599 patients exhibited preserved/moderately reduced LV function (Figure 1). The baseline characteristics of the patients are presented in Table I. The NYHA classification was more severe, and the brain natriuretic peptide (BNP) level was higher in patients with a reduced LV function. The use of CRTD and medical treatments for HF (betablockers, ACEI/ARB, spironolactone, and diuretics) was 


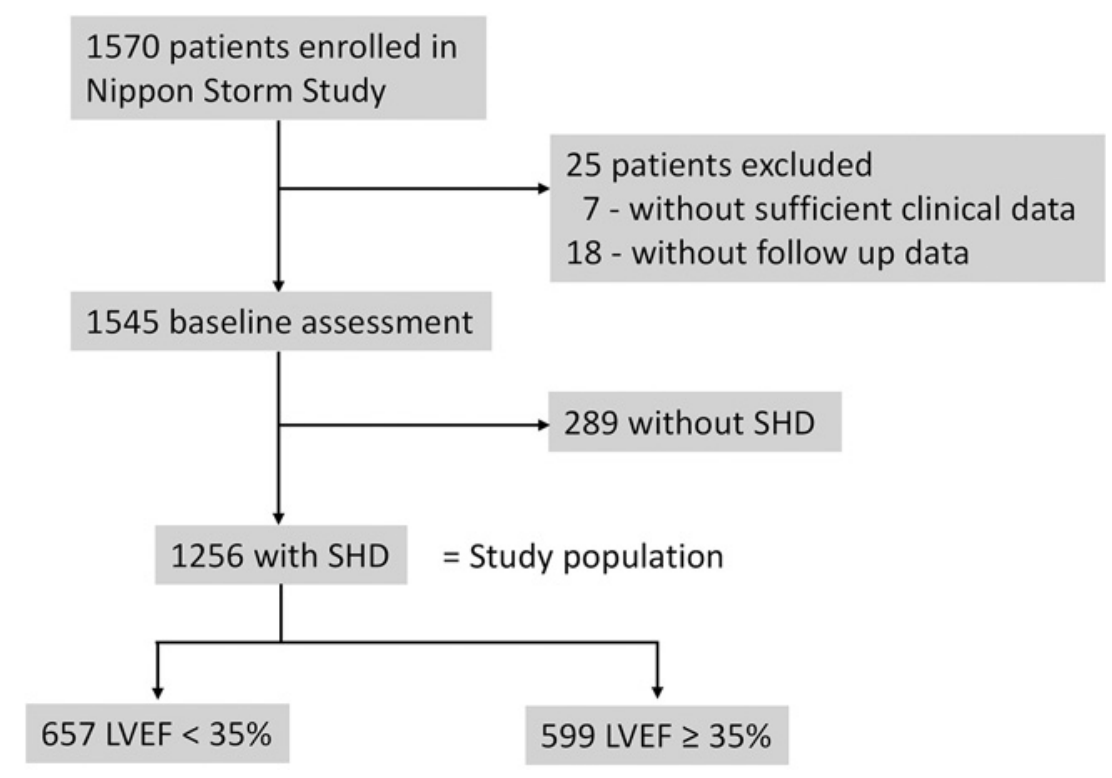

Figure 1. Flow chart of study design. SHD indicates structural heart disease; LV, left ventricular; and LVEF, left ventricular ejection fraction.

more frequent in patients with reduced LV function.

Incidence of ES: During the median follow-up period of 2.3 years (IQR: 1.9-2.7 years), ES occurred in 49 (7.5\%) and 36 patients $(6.0 \%)$ among those with reduced and preserved/moderately reduced LV function, respectively. There was no significant difference in the cumulative incidence of ES between the groups (log-rank $P=0.297$ ) (Figure 2). The incidence of ES among patients with ischemic heart disease was significantly higher in patients with a reduced $\mathrm{LV}$ function than in those with preserved or moderately reduced LV function (18/259 [6.9\%] versus 6/224 [2.7\%], $P=0.036$ ).

Risk of ES: Table II shows the respective ES risk factors for each group. The use of CRTD was significantly associated with a lower incidence of ES in patients with a reduced LV function (subdistribution hazard ratio [SHR] $0.44,95 \%$ confidence interval [CI]: $0.72-21.3$ ), but not in those with preserved/moderately reduced LV function $($ SHR $2.09,95 \%$ CI, 0.82-5.30) $(P$ for interaction $=$ $0.043)$. Non-ischemic heart disease and the use of diuretics were associated with ES in patients with preserved/ moderately reduced LV function, and these associations were more enhanced in these patients than in those with a reduced LV function (for non-ischemic heart disease, SHR 3.51 , 95\% CI: $1.46-8.47$ and SHR 1.23, 95\% CI: $0.72-$ 2.13, $P$ for interaction $=0.061$; for diuretics, SHR 2.49, 95\% CI: $1.25-4.98$ and SHR 1.95, 95\% CI: 1.19-3.17, $P$ for interaction $=0.002$ ) .

Mortality rate in patients with and without ES: During the median follow-up period of 2.3 years (IQR: 1.9-2.7), $10 / 49(20 \%)$ patients with ES and 80/608 patients (13\%) without ES died in the reduced LV function group, while $7 / 36$ (19\%) patients with ES and 38/563 patients (7\%) without ES died in the preserved/moderately reduced LV function group. The cumulative mortality rates across LV function and ES occurrence are shown in Figure 3. There was no significant difference in the mortality rate between patients with and without ES in the reduced $\mathrm{LV}$ function group (log-rank $P=0.154$ ) (Figure $3 \mathrm{~A}$ ). In contrast, mortality rate in patients with preserved/moderately reduced LV function was significantly higher in patients with ES than in those without (log-rank $P=0.006$ ) (Figure 3B).

Impact of ES on mortality: To estimate the prognostic impact of ES, we performed multivariate Cox regression analyses, which were adjusted for several combinations of relevant clinical variables (Table III). In all the models, ES was independently associated with all-cause death in all study patients (HR 3.14, 95\% CI: 1.83-5.40; HR 2.91, 95\% CI 1.69-5.00; HR 2.98, 95\% CI 1.73-5.13; HR 3.58, 95\% CI: 2.03-6.30 in models 1, 2, 3, and 4). HRs of ES for all-cause death were approximately 2-fold higher in the group with preserved or moderately reduced LV function compared to the group with a reduced function although the differences were not significant; HR 2.36, 95\% CI: $1.36-4.92$ and HR 5.02, 95\% CI: $2.20-11.46$ ( $P$ for interaction, 0.217 ) in model 1; HR 2.22, 95\% CI: $1.06-4.63$ and HR 4.41, 95\% CI 1.94-10.03 ( $P$ for interaction, 0.263 ) in model 2; HR 2.25, 95\% CI 1.07-4.69 and HR 4.26, 95\% CI 1.87-9.67 ( $P$ for interaction, 0.284) in model 3; HR 3.07, 95\% CI 1.40-6.74 and HR 5.04, 95\% CI 2.18-11.65 ( $P$ for interaction, 0.309 ) in model 4.

\section{Discussion}

The principal findings of the current study are as follows: (1) the incidence of ES was comparable between patients with reduced and preserved or moderately reduced LV function; (2) each patient group showed the respective risks of ES; CRTD was associated with a lower risk of ES in patients with a reduced LV function, whereas non-ischemic heart disease and the use of diuretics were associated with ES in patients with preserved/ 
Table I. Clinical Characteristics in Patients with Reduced and Preserve/Moderately Reduced LV Function

\begin{tabular}{|c|c|c|c|}
\hline & $\begin{array}{l}\text { Reduced LV function } \\
\quad n=657\end{array}$ & $\begin{array}{l}\text { Preserved/moderately } \\
\text { reduced LV function } \\
\quad n=599\end{array}$ & $P$ \\
\hline Age, years & $65 \pm 11$ & $65 \pm 13$ & 0.357 \\
\hline Male sex & $517(79 \%)$ & $433(72 \%)$ & 0.009 \\
\hline \multicolumn{4}{|l|}{ ICD indication } \\
\hline Primary prevention & $413(63 \%)$ & $223(37 \%)$ & \multirow[t]{2}{*}{$<0.001$} \\
\hline Secondary prevention & $244(37 \%)$ & $376(63 \%)$ & \\
\hline \multicolumn{4}{|l|}{ Structural heart disease } \\
\hline Ischemic & $259(39 \%)$ & $224(37 \%)$ & \multirow[t]{2}{*}{0.461} \\
\hline Non-ischemic & $398(61 \%)$ & $375(63 \%)$ & \\
\hline $\mathrm{HCM}$ & $26(4 \%)$ & $172(29 \%)$ & $<0.001$ \\
\hline DCM & $279(43 \%)$ & $69(12 \%)$ & $<0.001$ \\
\hline Sarcoidosis & $32(5 \%)$ & $30(5 \%)$ & 1.000 \\
\hline ARVC & $4(1 \%)$ & $27(5 \%)$ & $<0.001$ \\
\hline VHD & $21(3 \%)$ & $23(4 \%)$ & 0.543 \\
\hline CHD & $7(1 \%)$ & $14(2 \%)$ & 0.121 \\
\hline Others & $29(7 \%)$ & $40(7 \%)$ & 0.084 \\
\hline NYHA class & & & $<0.001$ \\
\hline I & $68(10 \%)$ & $290(48 \%)$ & \\
\hline II & $243(37 \%)$ & $238(40 \%)$ & \\
\hline$\geq \mathrm{III}$ & $346(53 \%)$ & $71(12 \%)$ & \\
\hline LVEF, $\%$ & $25 \pm 6$ & $52 \pm 13$ & $<0.001$ \\
\hline \multicolumn{4}{|l|}{ Electrocardiogram } \\
\hline Sinus rhythm & $504(77 \%)$ & $482(80 \%)$ & 0.114 \\
\hline $\mathrm{AF} / \mathrm{AFL}$ & $101(15 \%)$ & $72(12 \%)$ & 0.086 \\
\hline $\mathrm{HR}, \mathrm{bpm}$ & $70 \pm 15$ & $63 \pm 13$ & $<0.001$ \\
\hline QRS, ms & $142 \pm 36$ & $122 \pm 31$ & $<0.001$ \\
\hline QTc, ms & $476 \pm 53$ & $452 \pm 51$ & $<0.001$ \\
\hline \multicolumn{4}{|l|}{ Blood examination } \\
\hline $\mathrm{Hb}, \mathrm{g} / \mathrm{dL}$ & $13.0(11.0-14.0)$ & $13.0(12.0-14.0)$ & 0.071 \\
\hline $\mathrm{Cre}, \mathrm{mg} / \mathrm{dL}$ & $1.0(0.9-1.0)$ & $1.0(0.8-1.0)$ & $<0.001$ \\
\hline $\mathrm{BNP}, \mathrm{pg} / \mathrm{mL}$ & $379.0(174.8-757.8)$ & $183.0(78.0-419.0)$ & $<0.001$ \\
\hline \multicolumn{4}{|l|}{ Defibrillator } \\
\hline $\mathrm{ICD}$ & $247(38 \%)$ & $509(85 \%)$ & \multirow[t]{2}{*}{$<0.001$} \\
\hline CRTD & $410(62 \%)$ & $90(15 \%)$ & \\
\hline \multicolumn{4}{|l|}{ Comorbidities } \\
\hline Hypertension & $266(40 \%)$ & $283(47 \%)$ & 0.016 \\
\hline Diabetes mellitus & $203(31 \%)$ & $172(29 \%)$ & 0.398 \\
\hline Dyslipidemia & $220(34 \%)$ & $185(31 \%)$ & 0.325 \\
\hline Hyperuricemia & $113(17 \%)$ & $53(9 \%)$ & $<0.001$ \\
\hline \multicolumn{4}{|l|}{ Medications } \\
\hline Beta blocker & $493(75 \%)$ & $384(64 \%)$ & $<0.001$ \\
\hline ACEI/ARB & $452(69 \%)$ & $336(56 \%)$ & $<0.001$ \\
\hline Spironoractone & $299(46 \%)$ & $116(19 \%)$ & $<0.001$ \\
\hline Loop diuretics & $470(71 \%)$ & $234(39 \%)$ & $<0.001$ \\
\hline Statin & $205(31 \%)$ & $181(30 \%)$ & 0.714 \\
\hline Amiodarone & $271(41 \%)$ & $236(39 \%)$ & 0.527 \\
\hline Class 1 AAD & $25(4 \%)$ & $56(9 \%)$ & $<0.001$ \\
\hline
\end{tabular}

LV indicates left ventricular; ICD, implantable cardioverter-defibrillator; HCM, hypertrophic cardiomyopathy; DCM, dilated cardiomyopathy; ARVC, arrhythmogenic right ventricular cardiomyopathy; VHD, valvular heart disease; CHD, congenital heart disease; LVEF, left ventricular ejection fraction; NYHA, New York Heart Association; AF, atrial fibrillation; AFL, atrial flutter; EF, ejection fraction; CRTD, implantable cardioverter-defibrillator with resynchronization therapy; ACEI, angiotensin converting enzyme inhibitor; ARB, angiotensin II receptor blocker; and AAD, antiarrhythmic drug.

moderately reduced LV function, and these associations were more enhanced in these patients than in those with a reduced LV function. Finally, (3) the impact of ES on subsequent mortality was enhanced in patients with pre- served/moderately reduced LV function.

Incidence and risk of ES: When ICD was used for secondary prevention alone and CRT had not been introduced, ES incidences reportedly ranged between $10 \%$ and 


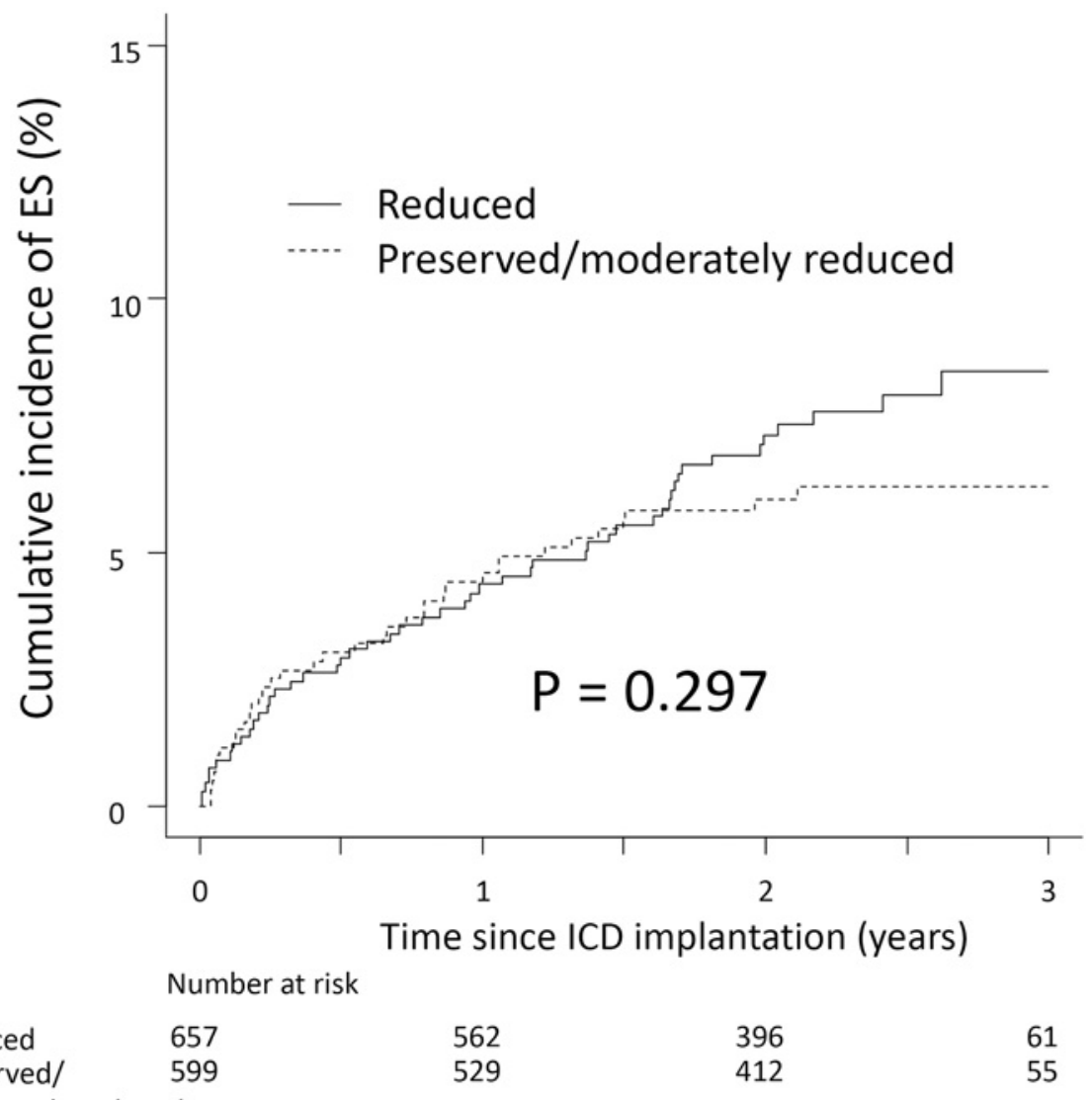

$\begin{array}{lllll}\text { Reduced } & 657 & 562 & 396 & 61 \\ \text { Preserved/ } & 599 & 529 & 412 & 55\end{array}$

moderately reduced

Figure 2. Cumulative incidence of electrical storm in patients with reduced and preserved/moderately reduced LV function. LV indicates left ventricular; ES, electrical storm; and ICD, implantable cardioverter-defibrillator.

Table II. Adjusted Hazard Ratios for Electrical Storm According to the Left Ventricular Function

\begin{tabular}{lccc}
\hline & $\begin{array}{c}\text { Reduced LV function } \\
\text { SHR }(95 \% \mathrm{CI})\end{array}$ & $\begin{array}{c}\text { Preserved/moderately reduced } \\
\text { LV function } \\
\text { SHR }(95 \% \mathrm{CI})\end{array}$ & $P$ for interaction \\
\hline CRTD & $0.44(0.24-0.82)$ & $2.09(0.82-5.30)$ & 0.043 \\
Non-ischemic cardiomyopathy & $1.23(0.72-2.13)$ & $3.51(1.46-8.47)$ & 0.061 \\
Diuretics & $1.95(1.19-3.17)$ & $2.49(1.25-4.98)$ & 0.002 \\
\hline
\end{tabular}

LV indicates left ventricular; SHR, subdistribution hazard ratio; CI, confidence interval; and other abbreviations as in Table I.

$28 \% .^{3,4,6,13)}$ After introducing ICD for primary prevention and CRTD, the incidence of ES decreased, generally ranging from $4 \%$ to $7 \% .^{5,8,11,14)}$ We are not aware of any prior reports of ES incidence in a specific population with preserved or moderately reduced EF. Therefore, we evaluated it in patients with an LVEF $\geq 35 \%$ and demonstrated that ES incidence in this population was $6 \%$, which is comparable to that of patients with an LVEF $<35 \%(7.5 \%)$ and aligns with recent reports $(4-7 \%)^{5,8,11,14)}$

Despite a comparable incidence of ES between the reduced and preserved/moderately reduced functional groups, the SHDs were quite different between them. Notably, patients with non-ischemic cardiomyopathy were more likely to have ES in the preserved LVEF group than in those with ischemic cardiomyopathy (Table II). Al- though some previous studies reported that reduced LVEF was associated with a higher incidence of $\mathrm{ES},{ }^{13,15)}$ this association has not been consistently confirmed. ${ }^{6,7)}$ This inconsistency may be partially explained by the different types of heart diseases included in the studies, that is, previous studies including a specific SHD, such as ischemia ${ }^{15)}$ or dilated cardiomyopathy, ${ }^{13)}$ which reported that a lower LVEF was a predictor of ES. The use of diuretics was also found to be a predictor of ES. The serum creatinine level and parameters related to HF, such as NYHA classification, LVEF, and medical treatments, were comparable between patients with and without ES, and no significant correlation was observed between the use of diuretics and those parameters. Conversely, we found that CRTD was related to a lower risk of ES in patients with a reduced 
A

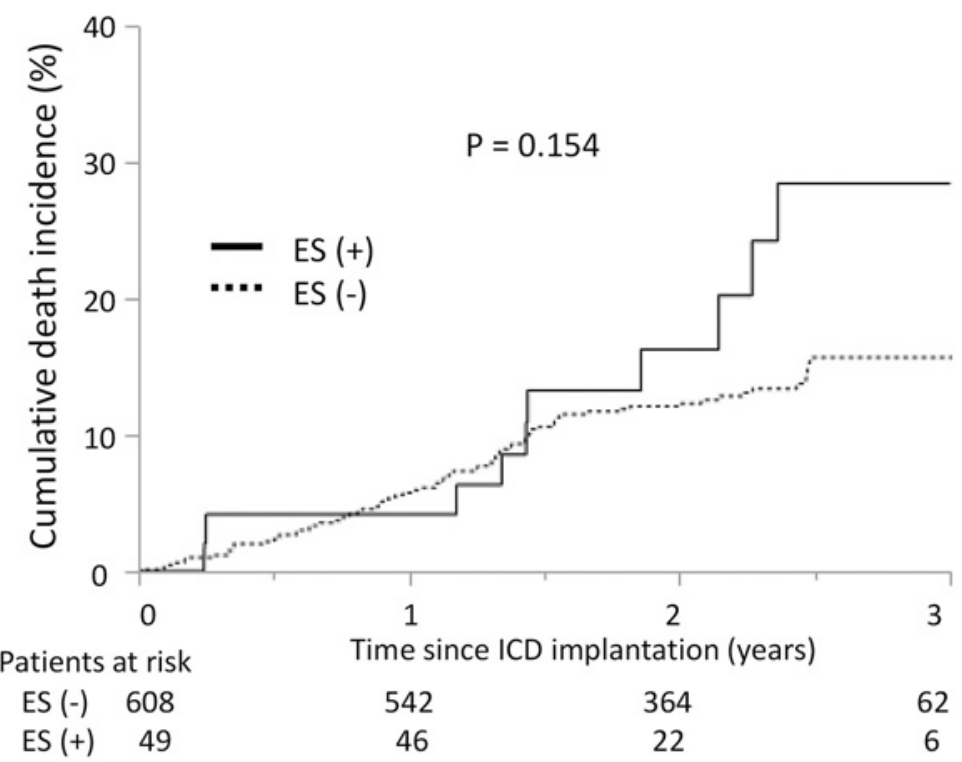

B

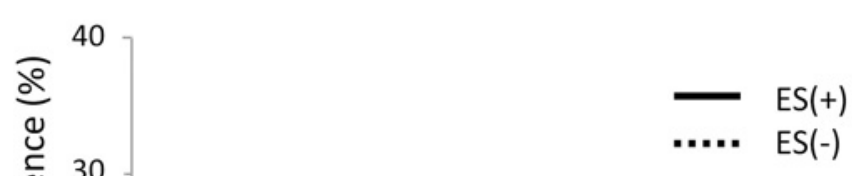

Figure 3. Cumulative incidence of all-cause death between patients with and without electrical storm (ES) in the groups with reduced left ventricular function (A) and preserved or moderately reduced left ventricular function $(\mathbf{B})$. ES indicates electrical storm; and ICD, implantable cardioverter-defibrillator.

Table III. Adjusted Hazard Ratios of Electrical Storm for All Cause Death

\begin{tabular}{|c|c|c|c|c|c|c|c|c|c|c|c|c|}
\hline & \multicolumn{3}{|c|}{ Model 1} & \multicolumn{3}{|c|}{ Model 2} & \multicolumn{3}{|c|}{ Model 3} & \multicolumn{3}{|c|}{ Model 4} \\
\hline & HR & $95 \%$ CI & $P$ & HR & $95 \% \mathrm{CI}$ & $P$ & HR & $95 \% \mathrm{CI}$ & $P$ & $\mathrm{HR}$ & $95 \% \mathrm{CI}$ & $P$ \\
\hline All patients & 3.14 & $1.83-5.40$ & $<0.001$ & 2.91 & $1.69-5.00$ & $<0.001$ & 2.98 & $1.73-5.13$ & $<0.001$ & 3.58 & $2.03-6.30$ & $<0.001$ \\
\hline Reduced LV function & 2.36 & $1.36-4.92$ & 0.021 & 2.22 & $1.06-4.63$ & 0.034 & 2.25 & $1.07-4.69$ & 0.032 & 3.07 & $1.40-6.74$ & 0.005 \\
\hline $\begin{array}{l}\text { Preserved/moderately } \\
\text { reduced LV function }\end{array}$ & 5.02 & $2.20-11.46$ & $<0.001$ & 4.41 & $1.94-10.03$ & $<0.001$ & 4.26 & $1.87-9.67$ & $<0.001$ & 5.04 & $2.18-11.65$ & $<0.001$ \\
\hline$P$ for interaction & & 0.217 & & & 0.263 & & & 0.284 & & & 0.309 & \\
\hline
\end{tabular}

All four models were adjusted for age and NYHA class $\geq$ III, and the following variables; sinus rhythm and heart rate in model 1, left ventricular function, LVEF and the use of CRTD in model 2; the use of beta-blockers and ACEIs or ARBs in model 3; creatinine and BNP in model 4. Abbreviations as in Table I. 
LV function. Guerra, et al. also reported that patients with CRTD had a lower incidence of ES than propensitymatched ICD patients. ${ }^{16)}$

Influence of ES on mortality: The influence of an increased risk of ES on mortality has been reported in several previous studies. ${ }^{5-8,13,14)}$ In addition, a recent metaanalysis reported that ES retained a significantly increased relative risk (RR) of 2.51 for death, even when compared with previous unclustered episodes of sustained VT. Furthermore, an increased RR of 3.34 was seen in these patients when compared with patients without any history of VT/VF. ${ }^{10)}$ The aforementioned prognostic influence of ES has been discussed in the entire ICD population (regardless of LVEF) in multiple single-center studies ${ }^{6,9,11,14)}$ and sub-studies of randomized controlled trials including patients with reduced LVEF $(\leq 35-40 \%)$. ${ }^{4,58}$ In the current study, we evaluated the prognostic impact of ES in patients with preserved/moderately reduced LV function ( $\mathrm{LVEF} \geq 35 \%$ ) and showed that ES was an independent mortality risk in those patients. Furthermore, the HR of ES for all-cause death was approximately twice as high in the group with preserved or moderately reduced LV function compared to the group with reduced function. In the group with reduced LV function, ES was also an independent mortality risk in multivariate Cox models whilst the estimated mortality rate by the Kaplan-Meier method was not significantly different between patients with and without ES. We consider this inconsistency was due to the difference in the statistical methods; in Cox models ES was considered as a time-dependent variable.

The comparison of clinical characteristics between the groups showed that patients with reduced function had more advanced HF conditions, higher BNP levels, and advanced renal dysfunction (Table I). The increase in these clinical characteristics was consistent with the higher mortality observed in these patients (Figure 3 ). Therefore, the disease condition in such patients might have attenuated the additive influence on mortality due to ES occurrence. Heterogeneous heart diseases were included in the group with a preserved/moderately reduced LV function, and we might have not included the possible confounders associated with mortality. However, our observation highlights that ES is associated with mortality in these patients and may suggest that ES is an important clinical manifestation associated with poor prognosis even in patients without severe LV dysfunction.

Limitations: This study has several limitations that should be addressed. First, the number of ES patients was relatively small, which limited the generalizability of the findings. Second, the Nippon Storm Study is a prospective, multicenter, observational study. Although we used a multivariate analysis and adjusted for confounding variables, potential unmeasured confounders may have biased the results. Third, the ES treatment was left to the discretion of the attending physician, which might have influenced the outcome. Finally, the mode of death was unclear in most cases.

\section{Conclusion}

Our observations suggest that the determinants of ES differ according to LV function and the impact of ES on mortality might be more enhanced in patients with preserved or moderately reduced LV function compared to those with reduced LV function. Appropriate management of ES is required to enhance the survival of patients at risk.

\section{Acknowledgments}

We gratefully acknowledge all 48 Japanese implantable cardiac shock device centers involved in this study and the office of the Japanese Heart Rhythm Society, especially Ms. Yoko Sato, for data collection. We also thank Drs. Naohiko Aihara and Keisuke Shioji for blindly confirming the E-Storm episodes based on intracardiac electrograms at the time of each event.

\section{Disclosure}

Conflicts of interest: IY reports grants from Health, Labour and Welfare Policy Research Grants, and speaker fees from Chugai Pharmaceutical Co, AstraZeneca, Japan Tobacco Pharmaceutical Division, and Nippon Shinyaku Co, outside the submitted work.

\section{References}

1. Al-Khatib SM, Stevenson WG, Ackerman MJ, et al. 2017 AHA/ ACC/HRS Guideline for Management of Patients With Ventricular Arrhythmias and the Prevention of Sudden Cardiac Death: A Report of the American College of Cardiology/American Heart Association Task Force on Clinical Practice Guidelines and the Heart Rhythm Society. Circulation 2018; 138: e272-391.

2. Proietti R, Sagone A. Electrical storm: Incidence, Prognosis and Therapy. Indian Pacing Electrophysiol J 2011; 11: 34-42.

3. Credner SC, Klingenheben T, Mauss O, Sticherling C, Hohnloser SH. Electrical storm in patients with transvenous implantable cardioverter-defibrillators: incidence, management and prognostic implications. J Am Coll Cardiol 1998; 32: 1909-15.

4. Exner DV, Pinski SL, Wyse DG, et al. Electrical storm presages nonsudden death: the antiarrhythmics versus implantable defibrillators (AVID) trial. Circulation 2001; 103: 2066-71.

5. Sesselberg HW, Moss AJ, McNitt S, et al. Ventricular arrhythmia storms in postinfarction patients with implantable defibrillators for primary prevention indications: a MADIT-II substudy. Heart Rhythm 2007; 4: 1395-402.

6. Hohnloser SH, Al-Khalidi HR, Pratt CM, et al. Electrical storm in patients with an implantable defibrillator: incidence, features, and preventive therapy: insights from a randomized trial. Eur Heart J 2006; 27: 3027-32.

7. Noda T, Kurita T, Nitta T, et al. Significant impact of electrical storm on mortality in patients with structural heart disease and an implantable cardiac defibrillator. Int J Cardiol 2018; 255: 8591.

8. Gasparini M, Lunati M, Landolina M, et al. Electrical storm in patients with biventricular implantable cardioverter defibrillator: incidence, predictors, and prognostic implications. Am Heart J 2008; 156: 847-54.

9. Guerra F, Flori M, Bonelli P, Patani F, Capucci A. Electrical storm and heart failure worsening in implantable cardiac defibrillator patients. Europace 2015; 17: 247-54.

10. Guerra F, Shkoza M, Scappini L, Flori M, Capucci A. Role of electrical storm as a mortality and morbidity risk factor and its clinical predictors: a meta-analysis. Europace 2014; 16: 347-53.

11. Nedios S, Darma A, Stevanello C, et al. Electrical storm in pa- 
tients with implantable cardioverter-defibrillator in the era of catheter ablation: Implications for better rhythm control. Heart Rhythm 2015; 12: 2419-25.

12. Pedersen CT, Kay GN, Kalman J, et al. EHRA/HRS/APHRS expert consensus on ventricular arrhythmias. Heart Rhythm 2014; 11: e166-96.

13. Bansch D, Bocker D, Brunn J, Weber M, Breithardt G, Block M. Clusters of ventricular tachycardias signify impaired survival in patients with idiopathic dilated cardiomyopathy and implantable cardioverter defibrillators. J Am Coll Cardiol 2000; 36: 566-73.
14. Nordbeck P, Seidl B, Fey B, Bauer WR, Ritter O. Effect of cardiac resynchronization therapy on the incidence of electrical storm. Int J Cardiol 2010; 143: 330-6.

15. Villacastin J, Almendral J, Arenal A, et al. Incidence and clinical significance of multiple consecutive, appropriate, highenergy discharges in patients with implanted cardioverterdefibrillators. Circulation 1996; 93: 753-62.

16. Guerra F, Palmisano P, Dell'Era G, et al. Cardiac resynchronization therapy and electrical storm: results of the OBSERVational registry on long-term outcome of ICD patients (OBSERVOICD). Europace 2018; 20: 979-85. 